\title{
Mouse spermatozoa with higher fertilization rates have thinner nuclei
}

\author{
Daisuke Mashiko ${ }^{\text {Corresp., }}{ }^{1}$, Masahito Ikawa ${ }^{2}$, Koichi Fujimoto ${ }^{\text {Corresp. } 1}$ \\ 1 Osaka University, Department of Biological Sciences, Toyonaka, Osaka, Japan \\ 2 Osaka University, Research Institute for Microbial Diseases, Suita, Osaka, Japan \\ Corresponding Authors: Daisuke Mashiko, Koichi Fujimoto \\ Email address: mashiko@bio.sci.osaka-u.ac.jp, fujimoto@bio.sci.osaka-u.ac.jp
}

Background. Although spermatozoa with normal morphology are assumed to have uniform fertilization ability, recent data show that even normal spermatozoa have considerable variation in their head shape which is associated with differences in fertilization ability. Appropriate quantitative indicators for good sperm morphology, however, remain unidentified.

Methods. Therefore, in an effort to identify such an indicator, we compared the nuclear contour of normal mouse spermatozoa by quantitative multivariate analysis using elliptic Fourier descriptors combined with principal component analysis. The spermatozoa were obtained from different strains and collection sites which have been shown to be associated with different fertilization abilities.

Results. We found that the head was 5.7\% thinner in spermatozoa from the B6D2F1 (BDF1) strain, known to have a higher fertilization rate, than in those from the C57BL/6N (B6N) strain, which has a lower fertilization rate. Moreover, zona-penetrated spermatozoa in the perivitelline space consistently had $5.4 \%$ thinner heads than those isolated from the epididymis before ejaculation. The aspect ratio, which represents the sperm head thinness, uniquely distinguished these sperm populations, confirming its validity as a morphological indicator.

Discussion. Because aspect ratio has also been shown to characterize human spermatozoa, this unique morphometric indicator might be applicable to compare normal spermatozoa among multiple patients, which will greatly facilitate and enhance current reproductive technologies. 


\section{Mouse Spermatozoa with Higher Fertilization Rates Have}

\section{Thinner Nuclei}

3

4 Daisuke Mashiko $^{1^{*}}$, Masahito Ikawa ${ }^{2}$, Koichi Fujimoto ${ }^{*}$

$6 \quad{ }^{1}$ Department of Biological Sciences, Osaka University, Japan.

$7 \quad{ }^{2}$ Research Institute for Microbial Diseases, Osaka University, Japan.

8 *Corresponding author

9 mashiko@bio.sci.osaka-u.ac.jp (DM), fujimoto@bio.sci.osaka-u.ac.jp (KF)

11 Running title: Sperm head morphology correlates with fertilization ability 


\section{Abstract}

13 Background. Although spermatozoa with normal morphology are assumed to have uniform

14 fertilization ability, recent data show that even normal spermatozoa have considerable variation

15 in their head shape which is associated with differences in fertilization ability. Appropriate

16 quantitative indicators for good sperm morphology, however, remain unidentified.

17 Methods. Therefore, in an effort to identify such an indicator, we compared the nuclear contour

18 of normal mouse spermatozoa by quantitative multivariate analysis using elliptic Fourier

19 descriptors combined with principal component analysis. The spermatozoa were obtained from

20 different strains and collection sites which have been shown to be associated with different

21 fertilization abilities.

22 Results. We found that the head was 5.7\% thinner in spermatozoa from the B6D2F1 (BDF1)

23 strain, known to have a higher fertilization rate, than in those from the C57BL/6N (B6N) strain,

24 which has a lower fertilization rate. Moreover, zona-penetrated spermatozoa in the perivitelline

25 space consistently had 5.4\% thinner heads than those isolated from the epididymis before

26 ejaculation. The aspect ratio, which represents the sperm head thinness, uniquely distinguished

27 these sperm populations, confirming its validity as a morphological indicator.

28 Discussion. Because aspect ratio has also been shown to characterize human spermatozoa, this

29 unique morphometric indicator might be applicable to compare normal spermatozoa among 
30 multiple patients, which will greatly facilitate and enhance current reproductive technologies. 


\section{Introduction}

32 Male infertility causes half of infertility cases in humans [Irvine, 1998]. To assess male

33 infertility, features of the population of spermatozoa in the semen that positively correlate with

34 fertilization ability, including the ratio of spermatozoa showing normal morphology and the number of motile spermatozoa [World Health Organization, 2010], have been used [Jouannet et. al., 1988; Toner et al., 1995; Eggert-Kruse et al., 1996; Coetzee et al.1998; Menkveld et al., 1990; Van Waart et al., 2001; Garrett et al., 2003; Liu et al., 2003]. One feature that can be assessed in a low-cost, high-throughput manner is sperm head morphology, which is affected by defects in nuclear structures (DNA [Gandini et al., 2000], chromosomes [Lee et al., 1996], chromatin [Dadoune et al., 1988; Devillard et al., 2002; Martin et al., 2003]). Most early studies on sperm fertilization indicators distinguished normal sperm from abnormal sperm that failed to

42 fertilize or resulted in embryos that spontaneously aborted [Fredricsson et al., 1977; Menkveld et al., 1990; Menkveld et al., 1991; Liu et al., 1992; Vilyana et al., 2017]. However, it would be beneficial to distinguish sperm with a better chance of fertilization among the population of normal sperm. For example, selection of good sperm morphology is beneficial when injecting a single spermatozoon into an ovum in the process known as intracytoplasmic sperm injection (ICSI) [Palermo et al., 1992] to resolve male infertility. It is unclear whether good sperm can be identified by indicators of infertility or whether they are represented by other morphologies, 
49 because normal sperm indicators that are quantitatively correlated with the success of

50 fertilization have not been well studied.

51 Even normal spermatozoa in semen are morphologically heterogeneous in their head

52 shape in mammals [Severa et al., 2010; Ramón et al., 2014]; human normal sperm morphologies have a range of 2.5-3.2 $\mu \mathrm{m}$ in width and 3.7-4.7 $\mu \mathrm{m}$ in length based on the WHO manual [World Health Organization, 2010]. Interestingly, semen containing an abundance of normal spermatozoa with elongated heads show higher fertilization ratio than that with rounded heads [Ramón et al., 2014]. This comparative morphology of sperm achieved some success as it was used to arbitrarily classify continuous variations of sperm head morphologies into several discrete categories which correlate fertilization ratio.

60 conventionally used indicators such as length, width, and angle are optimal. Moreover, they

61 could underestimate complex head contours by merely measuring distances between subjectively

62 selected points (Fig. S1 in Supplemental Information). Geometric morphometrics combining multivariate quantification of contour with systematic extraction of indicators have been

64 developed to circumvent such shortcomings, and this analysis has been applied to evaluate 65 morphological heterogeneities in mice [Oka et al., 2007], bovine [Ostermeier et al., 2001], 66 stallion [Severa et al., 2001], and human [Utsuno et al., 2013] sperm. The Elliptic Fourier 
67 descriptor (EFD) is a representative method to quantify any contour (closed curve) using

68 multiple ellipses [Severa et al., 2010, Kuhl et al, 1982; Iwata et al, 2002; Utsuno et al, 2013;

69 Beletti et al, 2005]. Principal component analysis (PCA) can provide the optimal indicators to

70 maximize variance among data (head contour of sperms) and compress multivariate information

71 into lower dimensions while retaining most of the original information. Therefore, a pipeline

72 integrating geometric morphometrics with comparative morphology among normal spermatozoa

73 with different fertilizability could objectively identify morphological indicators that correlate

74 with the success of fertilization.

To develop a pipeline that can determine indicators for high fertilizability, mice spermatozoa are a valuable model system because spermatozoa having known differences in fertilization rates (i.e., collection sites of spermatozoa and mouse strains) are available. The

hybrid BDF1 strain $(\mathrm{F} 1$ of inbred $\mathrm{B} 6 \mathrm{~N} \times \mathrm{DBA} / 2)$ has a higher fertilization ability than the inbred

C57BL/6N (B6N) strain [Sztein et al., 2000]. Spermatozoa that reach close proximity to the egg

80 (i.e., those in the cervical canal and in oviduct) are known to have higher fertilizability than those

81 collected just after ejaculation [Sztein et al., 2000; Cohen et al., 1974; Fischer et al., 1981;

82 Siddiquey et al., 1982]. Therefore, we applied geometric morphometrics to analyze sperm head contours within a population and between mouse strains and/or collection sites of spermatozoa.

84 To identify morphological indicators of highly fertile spermatozoa, and to develop a pipeline that 
85 might also be applicable to human normal sperm.

\section{Materials and Methods}

\section{Animals}

91 C57BL/6N (B6N) and BDF1 mice (12 weeks old) were obtained from SLC (JapanSLC, Inc.

92 Shizuoka, JP).

\section{Abnormality test}

previously [Touré et al., 2004; Wyrobek\&Bruce, 1975; Bruce et al., 1974; Watanabe 1991], we defined abnormal sperm as size outliers that showed a PC1 score $>0.55$ and largely lacked the hook-shaped heads common to most sperm.

\section{In vitro fertilization and spermatozoon collection}


103

104

105

106

107

108

109

110

111

112

113

114

115

116

117

118

119

120

chorionic gonadotropin (HCG, ASKA Pharmaceutical Co., Ltd. Tokyo, JP) $48 \mathrm{~h}$ after the PMSG

injection. The ovulated oocytes were collected from the oviducts $14 \mathrm{~h}$ after the HCG injection.

Cumulus-enclosed oocytes were placed in $100 \mu \mathrm{l}$ drops of TYH medium [Toyoda et al., 1971]

covered with paraffin oil (Nacalai Tesque, Kyoto, JP). The spermatozoa collected by mechanically-dissecting cauda epididymides $(\mathrm{N}=5)$ were placed in $100 \mu$ drop of TYH medium. After a 2-h incubation, the sperm suspension in TYH was added to the TYH drop containing eggs at a concentration of $5 \times 10^{5} \mathrm{sperm} / \mathrm{ml}$. After $2-8 \mathrm{~h}$ co-insemination of the spermatozoa and oocytes, the zona-bound spermatozoa were carefully removed using a holding needle. Because of the technical difficulty associated with collecting sperm in the zona pellucida of an oocyte by manipulation [Inoue et al, 2011], we obtained zona-penetrated spermatozoa using acidic Tyrode's solution after in vitro fertilization. Acidic Tyrode's solution treatment did not affect the sperm head aspect ratio. Zona pellucida was removed from the oocyte in $20 \mu 1$ acidic Tyrode's solution drops on a glass slide. The spermatozoa attached to the egg surface were removed by pipetting, and subsequently, the eggs were removed from the drops.

Epididymal spermatozoa were collected by dissecting the caput, corpus and cauda epididymides ( $\mathrm{N}=5$ for each experiment) and placed into $400 \mu$ phosphate buffered saline (PBS). Spermatozoa were collected from the oviduct and uterus by flushing out these structures with PBS $4 \mathrm{~h}$ after coitus. 


\section{Spermatozoon imaging and analysis}

123

The spermatozoa were coated onto a glass slide (Matsunami Glass, Osaka, JP) and

stained with $65 \mu \mathrm{M}$ Hoechst 33342 (Life Technologies, Carlsbad, CA). The slides were viewed

125

126

127

128

129

130

131

$132\left\{\begin{array}{l}X(t)=\sum_{n=1}^{N}\left(a_{n} \cos \frac{2 n \pi t}{T}+b_{n} \sin \frac{2 n \pi t}{T}\right) \\ Y(t)=\sum_{n=1}^{N}\left(c_{n} \sin \frac{2 n \pi t}{T}+d_{n} \cos \frac{2 n \pi t}{T}\right)\end{array}\right.$

$132\left\{\begin{array}{l}X(t)=\sum_{n=1}^{N}\left(a_{n} \cos \frac{2 n \pi t}{T}+b_{n} \sin \frac{2 n \pi t}{T}\right) \\ Y(t)=\sum_{n=1}^{N}\left(c_{n} \sin \frac{2 n \pi t}{T}+d_{n} \cos \frac{2 n \pi t}{T}\right)\end{array}\right.$

using an Olympus IX-70 fluorescence microscope with a 10× eyepiece and $100 \times$ objective lens

(Numerical apature is 1.4). The sperm head contours were derived from image binarization using the discriminant analysis method [Otsu 1975] in openCV (available also in ImageJ, http://imagej.nih.gov/ij/), which was customized using C programing language.

\section{Elliptic Fourier descriptors and principal component analysis}

Each sperm head was transferred to EFDs with two-dimensional coordinates given by:

where $n, N, t$, and $T$ denoted the harmonic number, the maximum harmonic number, the coefficients was provided as $4 N-3$ because normalization was carried out for the size and angle 
138

139

140

141

142

143

144

145

146

147

148

149

150

151

152

153

154

155

ratio. We approximated the contours of the heads of spermatozoa up to 20 ellipses $(\mathrm{N}=20)$, and

we performed the PCA on 77 parameters and the data reconstruction on the PC scores using

SHAPE (http://lbm.ab.a.u-tokyo.ac.jp/ iwata/shape/index.html) [Iwata et al., 2000].

\section{Statistics}

Kolmogorov-Smirnov test, Shapiro-Wilk test for checking normality, $F$-tests for checking homoscedasticity, $t$-tests and Steel-Dwass tests were performed using custom $\mathrm{R}$ programs. A $P$-value $>0.05$ was considered not significant (n.s.), whereas $P$-values $<0.05(*),<$ $0.01(* *)$, and $<0.001(* * *)$ were considered significant.

\section{Results}

\section{EFD and PCA revealed sperm head aspect ratio as unique fertility indicator.} In order to compare zona penetrated spermatozoa and ejaculated sperm, we focused on contour of the nucleus, which does not change by spontaneous acrosome reaction (See Figure S2A-C). To quantify the variations in sperm head morphology, we first collected spermatozoa (n = 179) from dissected B6N male cauda epididymides (Fig. 1A) and tracked the sperm head contour (Fig. 1B) by taking the pictures of each spermatozoon nucleus (Fig. S3). The tracked 
156

157

158

159

160

161

162

163

164

165

166

contour was sequentially input into a quantitative descriptor EFD method (see "Image analysis" and "Elliptic Fourier descriptors and principal component analysis" in Materials and Methods;

Fig. S4). To extract normal sperm characteristics, we subsequently performed PCA after subtracting out the abnormal spermatozoa (see "Abnormality test" in Materials and Methods; Fig. S5). Applying this protocol, we quantified the variation in head morphology of normal spermatozoa, as optimally separated into multiple principal components (PCs; Fig. S6), among B6N $(n=170$, Fig. 2A) and BDF1 spermatozoa $(n=163$, Fig. 2B). PC1 of the B6N spermatozoa highlighted variations in width (Figs. 2A and S6), whereas PC2 highlighted variations in the hook shape of the tip (Fig. S6).

To identify the PCs that correlate with fertilization ability, we focused on PC1, PC2, and

PC3, whose contribution rates were above $10 \%$ for $\mathrm{B} 6 \mathrm{~N}$ normal sperm $(49.8 \%, 19.3 \%$, and

12.7\%, respectively; Fig. S6). First, we identified the PCs that distinguished the BDF1 from the

B6N spermatozoa and the epididymis-isolated from the zona-penetrated B6N spermatozoa (see

"In vitro fertilization" in Materials and Methods). Regarding mouse strain, the BDF1 and B6N spermatozoa collected from the cauda epididymis showed differences in PC1, PC2, and PC3

(Figs. 2C and S7). However, regarding spermatozoa from different collection sites of spermatozoa, the epididymis-isolated and zona-penetrated B6N spermatozoa differed in PC1, but not in PC2 or PC3 (Figs. 2C and S7). Thus, PC1 uniquely distinguished spermatozoa between 
174

175

176

177

178

179

180

181

182

183

184 185

186

187

188

189

190

191

mouse strains and between collection sites of spermatozoa.

Next, we tried to identify the morphological parameters contributing to PC1. The width of the sperm head, defined as the longest part of the head perpendicular to its length, seemed to be highlighted in PC1 (Fig. 2A), but the error in width measurement was large. On the other hand, the major and minor axes of the ellipse representing the lowest mode of the sperm head EFD were almost precisely overlapped with the antero-posterior (length) and dorso-ventral (width) axes of the sperm head, respectively (Figs. 3A and S8A). Thus, we used these major and minor axes to calculate the aspect ratios (minor axis divided by the major axis, equivalent to $d_{1}$ in Eq. (1); Fig. 3A). We found that the sperm head aspect ratios of BDF1 spermatozoa $(\mathrm{n}=330)$ were reduced compared with those of B6N spermatozoa (Fig. 3B; $\mathrm{n}=298$; one-tailed $t$-test, $P=$ $\left.2.1 \times 10^{-16}\right)$, whereas the sperm head areas were not significantly different between the two groups (Fig. S9A, B; $\mathrm{n}=687$ for BDF1; $\mathrm{n}=465$ for B6N; two-tailed $t$-test, $P=0.53$ ). Moreover, the factor loading of the aspect ratio on $\mathrm{PCl}$, which was proportional to the correlation coefficient with PC1, was more than 2-fold higher than that of any of the other parameters of the EFDs (x-coordinate in Fig. 3C). Thus, by combining PCA and EFDs, we established a quantitative evaluation pipeline of multi-dimensional morphological variation, and using this pipeline, we identified a low aspect ratio, i.e., a thin sperm head, as a unique and optimal morphological indicator of the fertilization ability of spermatozoa. 
The sperm head aspect ratio decreased during the progression of fertilization.

spermatozoa ( $\mathrm{n}=118$, green in Fig. 4) with zona-penetrated B6N spermatozoa isolated from the perivitelline space (PVS) $(n=133$, red in Fig. 4 and Fig. S10). The sperm head aspect ratio of zona penetrated spermatozoa was significantly smaller than that of cauda spermatozoa (Fig. 4; unpaired, one-tailed $t$-test, $P=2.2 \times 10^{-16}$ ), further confirming the validity of the sperm head aspect ratio as a fertilization indicator. Moreover, the $\mathrm{B} 6 \mathrm{~N}$ zona penetrated spermatozoa had an aspect ratio that was similar to that of BDF1 cauda epididymis-isolated spermatozoa (Fig. 4, Kolmogorov-Smirnov test, $P=0.57$ ), which have a higher fertilization rate than $\mathrm{B} 6 \mathrm{~N}$ epididymis-isolated spermatozoa. Importantly, the mean aspect ratio of spermatozoa decreased throughout the progression of fertilization, and it was consistently negatively correlated with the fertilization ability of the spermatozoa. To investigate whether the sperm head aspect ratio decreased during sperm maturation in male mice prior to ejaculation, we collected spermatozoa aspect ratio of spermatozoa from the caput $(n=69$, purple box in Fig. 4) was 3.0\% larger than that from the corpus $(\mathrm{n}=85$, sky blue in Fig. 4; Steel-Dwass test, $P=0.01)$ and $2.6 \%$ larger than 
210 aspect ratios of spermatozoa from the corpus and cauda were not different from each other

211 (Steel-Dwass test, $P=0.98$ ). In addition, the minor axis length of the ellipse was decreased by

$2123.1 \%$ from the caput to the cauda (Fig. S8B; two-tailed $t$-test, $P=0.015$ ), whereas the major axis

213 length was not significantly different between those groups (two-tailed $t$-test, $P=0.58$ ). Similar

214 to the aspect ratio means, the coefficients of variation (i.e. SD normalized by mean) of the aspect

215 ratios of $\mathrm{B} 6 \mathrm{~N}$ spermatozoa continuously decreased from the caput $(8.4 \%)$, to the corpus $(6.0 \%)$,

216 to the cauda $(5.3 \%)$.

$218=65$; Fig. 1A) of females post-coitus, and we did not observe a difference in mean aspect ratio

219 between these two locations (unpaired two-tailed $t$-test, $P=0.22$ ). Moreover, to address whether

220 the acrosome reaction [Dan 1952] causes the decreased aspect ratio of the zona-penetrated

221 spermatozoa, we analyzed sperm morphology before and after the acrosome reaction by using

222 transgenic Acr-EGFP (EGFP fused to the acrosome migrating signal; Fig. S2) mice and found

223 that the acrosome reaction did not affect the aspect ratio in sperm nucleous. Taken together,

224 these data demonstrate that the aspect ratio of the sperm head did not change during entry of

225 spermatozoa into the oviduct, but decreased during spermatozoon zona penetration. 


\section{Discussion}

228 Possible mechanisms of the decreasing sperm head aspect ratio throughout

\section{9 the fertilization process}

During spermatozoon maturation in the epididymides of $\mathrm{B} 6 \mathrm{~N}$ male mice, we found major morphological changes including decreases in head aspect ratios and minor axis lengths of the normal spermatozoa (Figs. 4 and S8B). These decreases are likely due to structural changes that take place inside the sperm nucleus, which largely occupies the sperm head. During spermatogenesis in the testis, the DNA packaging histones are replaced by protamines, which more densely package every $50-60 \mathrm{~kb}$ of DNA into a toroid (donut) shape [Braun \& Robert 2001]. In the caput, the toroids are subsequently cross-linked by disulfide (SS) bonds, resulting in further DNA compaction [Bedford \& Calvin 1974], which is consistent with our observed decrease in the sperm head minor axis length and aspect ratio in the caput-isolated spermatozoa. Thus, we hypothesize that the decreased aspect ratio in maturing spermatozoa from the epididymis is caused by the formation of SS bonds, which can be tested using dithiothreitol to

241 reduce the SS bonds [Bedford \& Calvin 1974] and determine whether they are indeed

242 responsible for these morphological changes. 
245 and oviduct) [Krzanowska, 1974; Anne \& Mary 1984]. Here, we showed that the normal

246 spermatozoa isolated from the uteri and oviducts after coitus had similar sperm head aspect ratios

247 (Fig. 4), suggesting that UTJ plays a role in eliminating the abnormal spermatozoa but not in

248 altering normal sperm morphology. The decreases in sperm head aspect ratios that we observed

249 during zona penetration (Fig. 4) could be due to the selection of a fractional sperm population

250 [Utsuno et al., 2013; Beletti et al., 2005] or to the deformation of individual sperm. Sperm

251 deformation has been attributed to the acrosome reaction [Dan 1952], which, in our case, we

252 showed to be unlikely (Fig. S2), or to the mechanical force loaded at the time of the zona

253 penetration. Thus, determining whether sperm subpopulation selection or mechanical

254 deformation occurs during zona penetration, using methods such as time-lapse imaging and/or

255 measuring the yield stress on the sperm head during zona penetration, will be important avenues

256 for future examination.

Strategies for identifying the most fertile spermatozoa and implication for therapeutic applications suggests that spermatozoon morphology can be used as a strategy to screen mouse spermatozoa suitable for fertilization. Here, we showed that populations of spermatozoa with higher 
263 fertilization abilities (BDF1 cauda and B6N zona-penetrated in Fig. 4) had smaller aspect ratios,

264 i.e., thinner heads, than other populations. The generality of this correlation should be further

265 validated by applying our morphometry to zona-penetrated spermatozoa of other strains whose

266 fertilization rates are well known, including DBA/2, BALB/c, 129S3/SvIm, and FVB/N.

267 Moreover, even in spermatozoon populations with a large mean aspect ratio, there could be

268 subpopulations with smaller aspect ratios and increased fertilization abilities. For instance, in our

269 study, we observed a fraction of B6N cauda spermatozoa that morphologically overlapped with

270 BDF1 cauda spermatozoa and B6N zona-penetrated spermatozoa, which have higher fertilization

271 abilities ( $\mathrm{PC} 1=0.3-0.4$ in Fig. 2C). Thus, evaluating whether these morphologically unique

272 subpopulations have correspondingly altered fertilization abilities will be another important

273 avenue for future examination that will ultimately enable us to screen heterogeneous

274 spermatozoon populations to identify spermatozoa that are suitable for fertilization by their

275 morphological characteristics, such as their sperm head aspect ratio.

276

277 Applicability to human sperm

278 The correlation between aspect ratio and the success of fertilization in mice is consistent

279 with a study showing that elongated sperm is more favorable for fertilization than rounded sperm

280 [Ramón et al., 2014]. Human spermatozoa have been represented mainly by ellipses using 
281 Elliptic Fourier descriptors [Utsuno et al., 2013]. Therefore, the present pipeline, which 282 integrates geometric morphometrics with comparative morphology (Figs. 2 and 3), could be 283 applied under microscope in clinic laboratories, as long as edge detection of multiple sperms is 284 performed with sufficiently low error rate (Fig. 1B) so as to quantitatively examine the 285 correlation between the aspect ratio normal sperm and fertilization success among human 286 patients. This would greatly facilitate and enhance current reproductive technologies in an 287 objective, high-throughput manner.

\section{Acknowledgements}

291 We thank S. Nishioka and Y. Esaki for preparing the zygotes; members of the theoretical biology

292 laboratory for constructive criticisms; S.A.M. Young and T. Yao for critical reading of the manuscript.

\section{References}

1. Irvine, D. S., 1998, Epidemiology and aetiology of male infertility. Human Reproduction, 13 
2. World Health Organization, 2010, WHO Laboratory Manual for the Examination of Human

Semen and Sperm-Cervical Mucus Interaction.

300

3. Jouannet, P., Ducot, B., Feneux, D., and Spira, A., 1988, Male factors and the likelihood of

pregnancy in infertile couples. I. Study of sperm characteristics. International journal of

andrology, 11(5), 379-394. DOI (http://dx.doi.org/10.1111/j.1365-2605.1988.tb01011.x)

4. Toner, J. P., Mossad, H., Grow, D. R., Morshedi, M., Swanson, R. J., and Oehninger, S., 1995,

Value of sperm morphology assessed by strict criteria for prediction of the outcome of artificial

(intrauterine) insemination. Andrologia, 27(3), 143-148. DOI (http://dx.doi.org/10.1111/j.1439-

306

0272.1995.tb01085.x)

307

5. Eggert-Kruse, W., Schwarz, H., Rohr, G., Demirakca, T., Tilgen, W., and Runnebaum, B.,

1996, Sperm morphology assessment using strict criteria and male fertility under in-vivo

conditions

of

conception. Human

reproduction, 11(1),

139-146.

DOI

(http://dx.doi.org/10.1093/oxfordjournals.humrep.a019007)

311

6. Coetzee, K., Kruger, T. F., and Lombard, C. J., 1998, Predictive value of normal sperm

morphology: a structured literature review. Human Reproduction Update, 4(1), 73-82. DOI

(http://dx.doi.org/10.1093/humupd/4.1.73)

7. Menkveld, R., Stander, F. S., Kruger, T. F., and van Zyl, J. A., 1990, The evaluation of 
316

317

318

319

320

321

322

323

324

325

326

327

328

329

330

331

332

into

mouse

oocytes. Human

Reproduction, 11(9),

1942-1946.

Reproduction, 5(5), 586-592. DOI (http://dx.doi.org/10.1093/oxfordjournals.humrep.a137150)

Human Reproduction Update, 7(5), 495-500. DOI (http://dx.doi.org/10.1093/humupd/7.5.495)

9. Garrett, C., Liu, D. Y., Clarke, G. N., Rushford, D. D., and Baker, H. W. G., 2003, Automated

semen analysis:'zona pellucida preferred'sperm morphometry and straight-line velocity are

related to pregnancy rate in subfertile couples. Human Reproduction, 18(8), 1643-1649. DOI

(http://dx.doi.org/10.1093/humrep/deg306)

10. Liu, D. Y., Garrett, C., \& Baker, H. W. G., 2003, Low proportions of sperm can bind to the zona pellucida of human oocytes. Human Reproduction, 18(11), 2382-2389. DOI 
334 13. Dadoune, J. P., Mayaux, M. J., \& Guihard-Moscato, M. L., 1988, Correlation between

335 defects in chromatin condensation of human spermatozoa stained by aniline blue and semen

336 characteristics. Andrologia, 20(3), 211-217. $\quad$ DOI $\quad$ (http://dx.doi.org/10.1111/j.1439-

337 0272.1988.tb01058.x)

338 14. Devillard, F., Metzler-Guillemain, C., Pelletier, R., DeRobertis, C., Bergues, U., Hennebicq,

339 S. \& Rousseaux, S., 2002, Polyploidy in large-headed sperm: FISH study of three cases. Human

340 Reproduction, 17(5), 1292-1298. DOI (http://dx.doi.org/10.1093/humrep/17.5.1292)

341 15. Martin, R. H., Rademaker, A. W., Greene, C., Ko, E., Hoang, T., Barclay, L., \& Chernos, J.,

342 2003, A comparison of the frequency of sperm chromosome abnormalities in men with mild,

343 moderate, and severe oligozoospermia. Biology of reproduction, 69(2), 535-539.

344 16. Fredricsson B, Björk G. 1977, Morphology of postcoital spermatozoa in the cervical

345 secretion and its clinical significance. Fertility and Sterility, 28:841-845. DOI

346 (http://dx.doi.org/10.1016/S0015-0282(16)42738-X)

347 17. Menkveld, R., Stander, F. S., Kruger, T. F., and van Zyl, J. 1990, A. The evaluation of

348 morphological characteristics of human spermatozoa according to stricter criteria. Human

349 Reproduction, 5(5), 586-592. DOI (http://dx.doi.org/10.1093/oxfordjournals.humrep.a137150)

350 18. Menkveld, R., Franken, D. R., Kruger, T. F., Oehninger, S., and Hodgen, G. D., 1991, Sperm

351 selection capacity of the human zona pellucida. Molecular reproduction and development, 30(4), 
352

353

354

355

356

357

358

359

360

361

362

363

364

346-352. DOI (http://dx.doi.org/10.1002/mrd.1080300409)

19. Liu, D. Y., and Baker, H. W., 1992, Tests of human sperm function and fertilization in vitro.

Fertility and sterility, 58(3), 465-483.

20. Vilyana G., Dimitar P., Efrosini T., Todor C., 2017, Relation between morphological defects in human spermatozoa and spontaneous abortions in patients undergoing ICSI, Annuaire de l’Université de Sofia “St. Kliment Ohridski” Faculte de Biologie, 102(4), 186-193.

21. Palermo, G., Joris, H., Devroey, P., \& Van Steirteghem, A. C., 1992, Pregnancies after intracytoplasmic injection of single spermatozoon into an oocyte. The Lancet, 340(8810), 17-18. DOI (http://dx.doi.org/10.1016/0140-6736(92)92425-F)

22. Severa, L., Máchal, L., Švábová, L., and Mamica, O., 2010, Evaluation of shape variability of stallion sperm heads by means of image analysis and Fourier descriptors. Animal reproduction science, 119(1), 50-55. DOI (http://dx.doi.org/10.1016/j.anireprosci.2009.12.007)

23. Ramón, M., Jiménez Rabadán, P., García-Álvarez, O., Maroto Morales, A., Soler, A. J., Fernández Santos, M. R. \& Garde, J. J., 2014, Understanding sperm heterogeneity: biological and practical implications. Reproduction in Domestic Animals, 49(s4), 30-36. DOI (http://dx.doi.org/10.1111/rda.12404) 
368

369

370

371

372

373

374

375

376

377

378

379

380

381

382

383

384

385

24. Kuhl, F. P., and C. R. Giardina., 1982, Elliptic Fourier features of a closed contour.

Computer Graphics and Image Processing, 18: 236-258. DOI (http://dx.doi.org/10.1016/0146$664 X(82) 90034-X)$

25. Iwata, H., and Y. Ukai., 2002, SHAPE: A computer program package for quantitative evaluation of biological shapes based on elliptic Fourier descriptors. Journal of Heredity, 93:

384-385. DOI (http://dx.doi.org/10.1093/jhered/93.5.384)

26. Utsuno, H., Oka, K., Yamamoto, A., \& Shiozawa, T., 2013, Evaluation of sperm head shape at high magnification revealed correlation of sperm DNA fragmentation with aberrant head ellipticity and angularity. Fertility and sterility, 99(6), 1573-1580. DOI (http://dx.doi.org/10.1016/j.fertnstert.2013.01.100)

27. Beletti, M. E., da Fontoura Costa, L., \& Viana, M. P., 2005, A comparison of morphometric characteristics of sperm from fertile Bos taurus and Bos indicus bulls in Brazil. Animal reproduction science, 85(1), 105-116. DOI (http://dx.doi.org/10.1016/j.anireprosci.2004.04.019)

28. Oka A, Aoto T, Totsuka Y, Takahashi R, Ueda M, Mita A, \& Moriwaki, K. 2007, Disruption of genetic interaction between two autosomal regions and the $\mathrm{X}$ chromosome causes reproductive isolation between mouse strains derived from different subspecies. Genetics;175:185-97. DOI (http://dx.doi.org/10.1534/genetics.106.062976)

29. Ostermeier GC, Sargeant GA, Yandell TBS, Parrish JJ., 2001, Measurement of sperm 
386 nuclear shape using Fourier harmonic amplitudes. J Androl;22:584-94.

387 30. Severa L, M_achal L, Sv_abov_a L, Mamica O., 2010, Evaluation of shape variability of 388 stallion sperm heads by means of image analysis and Fourier descriptors. Anim Reprod 389 Sci;119:50-5. DOI (http://dx.doi.org/10.1016/j.anireprosci.2009.12.007)

31. Sztein, J. M., Farley, J. S., and Mobraaten, L. E., 2000, In vitro fertilization with cryopreserved inbred mouse sperm. Biology of Reproduction. 63(6), 1774-1780.

32. Cohen, J. and McNaughton, D., 1974, Spermatozoa: the probable selection of a small population by the genital tract of the female rabbit. J. Reprod. Fert. 39, 297-310. DOI (http://dx.doi.org/10.1530/jrf.0.0390297)

33. Fischer B, Adams CE., 1981, Fertilization following mixed insemination with 'cervixselected' and 'unselected' spermatozoa in the rabbit. J Reprod Fertil, 62:337-343. DOI (http://dx.doi.org/10.1530/jrf.0.0620337)

34. Siddiquey AK, Cohen J., 1982, In-vitro fertilization in the mouse and the relevance of different sperm/egg concentrations and volumes. $J$ Reprod Fertil;;66:237-242. DOI

401 35. Touré, A., Szot, M., Mahadevaiah, S. K., Rattigan, Á., Ojarikre, O. A., and Burgoyne, P. S., 402 2004, A new deletion of the mouse Y chromosome long arm associated with the loss of Ssty 
403 404 (http://dx.doi.org/10.1534/genetics.166.2.901)

expression, abnormal sperm development and sterility. Genetics, 166(2), 901-912. DOI

36. Wyrobek, A. J., and W. R. Bruce., 1975, Chemical induction of sperm abnormalities in mice.

406

Proceedings of the National Academy of Sciences, 72.11. 4425-4429. DOI (http://dx.doi.org/10.1073/pnas.72.11.4425)

37. Bruce, W. R., R. Furrer, and A. J. Wyrobek., 1974. Abnormalities in the shape of murine 409 sperm after acute testicular X-irradiation. Mutation Research/Fundamental and Molecular Mechanisms of Mutagenesis, 23.3, 381-386. (http://dx.doi.org/10.1016/0027-5107(74)90112-2)

38. Watanabe, Toshiaki, and Akira Endo., 1991, Effects of selenium deficiency on sperm morphology and spermatocyte chromosomes in mice. Mutation Research Letters, 262.2, 93-99.

DOI (http://dx.doi.org/10.1016/0165-7992(91)90113-I)

39. Toyoda, Y., Yokoyama, M. and Hoshi, T., 1971, Studies on the fertilization of mouse eggs in vitro. Jpn J Anim Reprod, 16, 147-151. DOI (http://dx.doi.org/10.1262/jrd1955.16.152)

40. Inoue, N., Satouh, Y., Ikawa, M., Okabe, M., and Yanagimachi, R., 2011, Acrosome-reacted mouse spermatozoa recovered from the perivitelline space can fertilize other eggs. Proceedings

418 of the National Academy of Sciences, 108(50), 20008-20011. DOI 419 (http://dx.doi.org/10.1073/pnas.1116965108) 
420 41. Otsu, N., 1975, A threshold selection method from gray-level histograms. Automatica

421 11(285-296), 23-27. DOI (http://dx.doi.org/10.1109/TSMC.1979.4310076)

422 42. DAN, J. C., 1952, Studies on the acrosome. I. Reaction to egg-water and other stimuli. Biol.

423 Bull. 103, 54-66 DOI (http://dx.doi.org/10.2307/1538405)

424 43. Braun, Robert E., 2001, Packaging paternal chromosomes with protamine. Nature genetics,

28.1, 10-12. DOI (http://dx.doi.org/10.1038/ng0501-10)

44. Bedford, J.M. \& Calvin, H.I., 1974, The occurrence and possible functional significance of -

SS- crosslinks in sperm heads, with particular reference to eutherian mammals. Exp. Zool., $188,137-55$

45. Krzanowska, Halina. ,1974, The passage of abnormal spermatozoa through the uterotubal

430 junction of the mouse. Journal of reproduction and fertility, 38.1, 81-90. DOI

431 (http://dx.doi.org/10.1530/jrf.0.0380081)

432 46. Anne Nestor and Mary Ann Handel., 1984, The Transport of Morphologically Abnormal 433 Sperm in the Female Reproductive Tract of Mice. Gamete Research, 10:11Y-125. DOI 434 (http://dx.doi.org/10.1002/mrd.1120100204)

435 47. Nakanishi, T., Isotani, A., Yamaguchi, R., Ikawa, M., Baba, T., Suarez, S. S. \& Okabe, M., 436 2004, Selective passage through the uterotubal junction of sperm from a mixed population 
437 produced by chimeras of calmegin-knockout and wild-type male mice. Biology of 438 reproduction, 71(3), 959-965. DOI (http://dx.doi.org/10.1095/biolreprod.104.028647) 


\section{Figure 1}

Schematic diagrams illustrate the methods of spermatozoon isolation and sperm head contour extraction.

(A) The male and female reproductive tracts. Spermatozoa were collected from the epididymis of males or the oviducts or uteri of females. Zona-penetrated spermatozoa were isolated following in vitro fertilization. The red arrow shows the zona pellucida. (B) The shapes of the sperm heads were visualized using Hoechst 33342 to stain the nuclei. Image binarization and extraction of the contours were subsequently performed. 
A epididymis
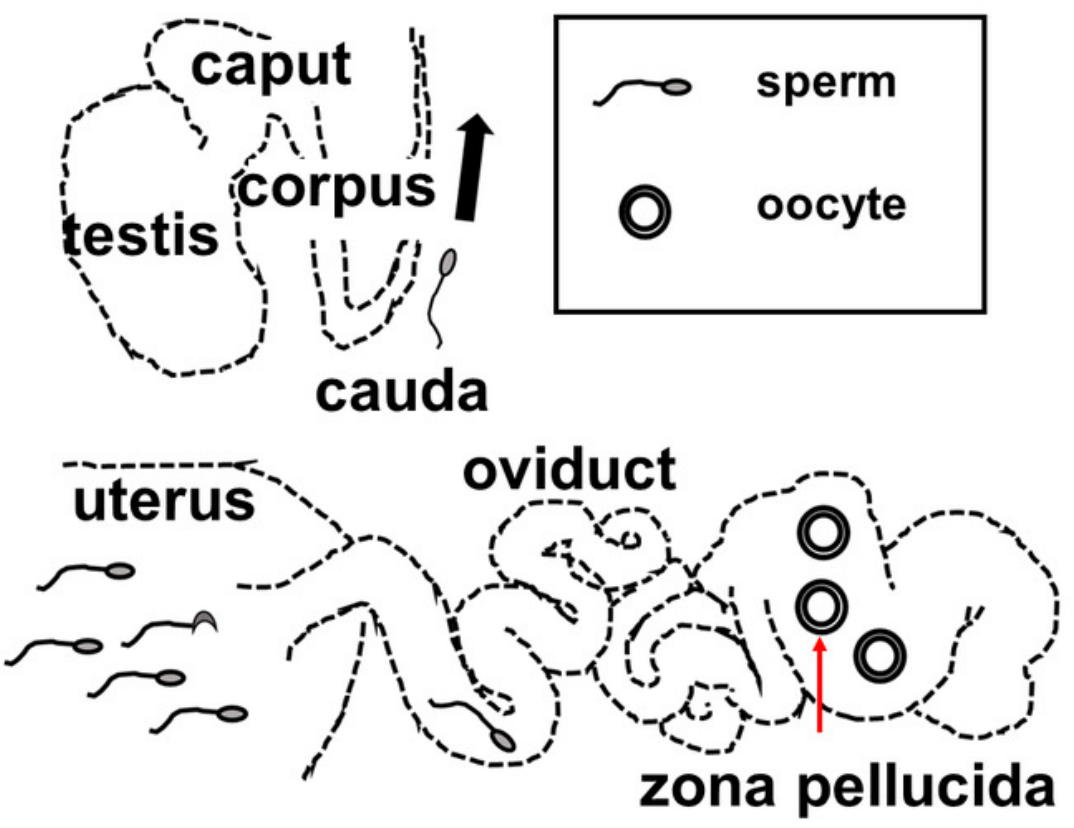

B
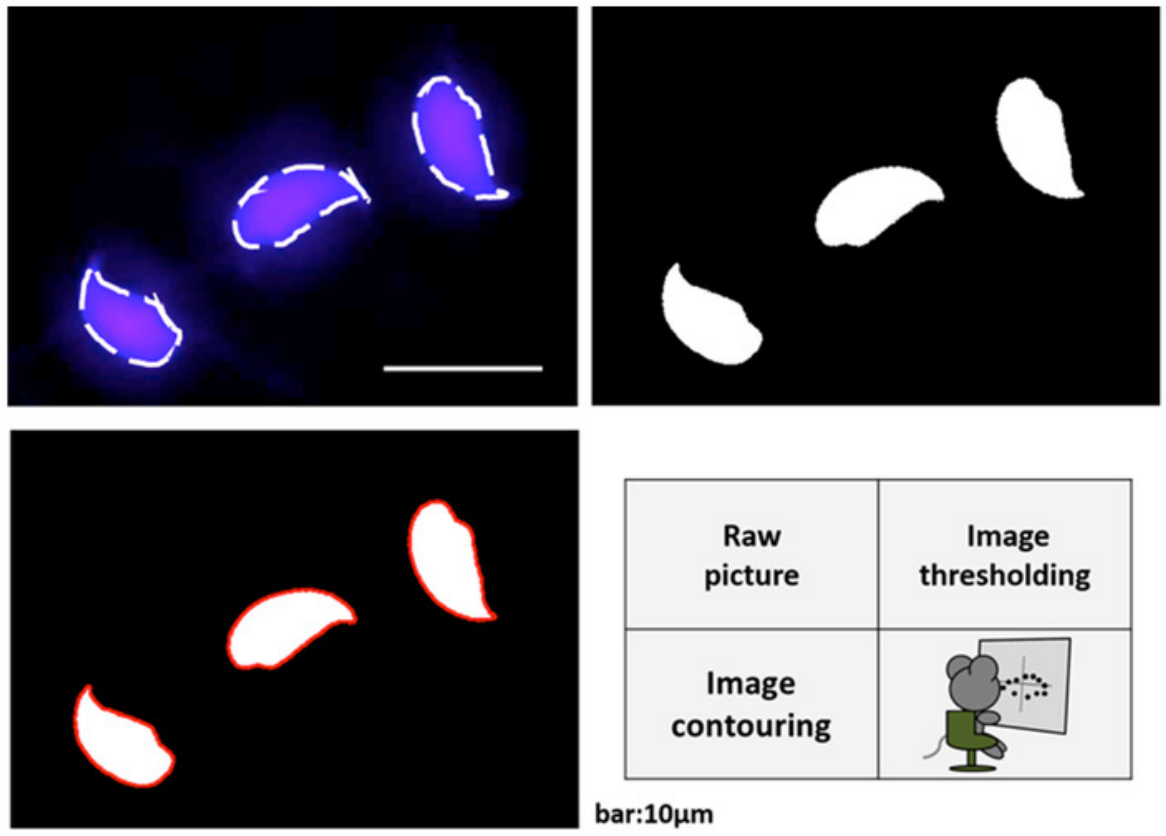

bar: $10 \mu \mathrm{m}$ 


\section{Figure 2}

The first principal component distinguished variation in normal spermatozoa among strains and fertilization stages.

Minus 2 standard deviation (SD), mean, and +2 SD sperm head contours of the first principal component (PC1) are shown from (A) B6N and (B) BDF1 mouse epididymis-isolated spermatozoa. The arrowhead shows the region of increased variation. (C) A scatter plot and the associated density curves (outside of the $x$ - and $y$-axis) of the PC scores of B6N zonapenetrated spermatozoa (red), and B6N (green) and BDF1 (black) cauda epididymis-isolated spermatozoa. The distribution of each group partially overlapped at $\mathrm{PC1}=0.3-0.4$. The eigenvectors of the PCs were derived from the principal component analysis (PCA) of B6N epididymis-isolated spermatozoa. 
A

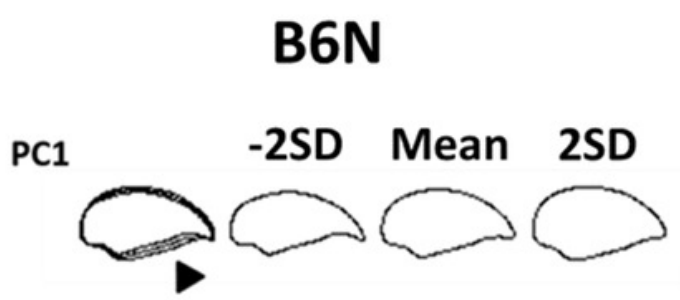

B

BDF1

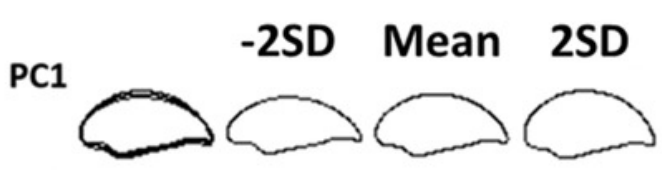

C

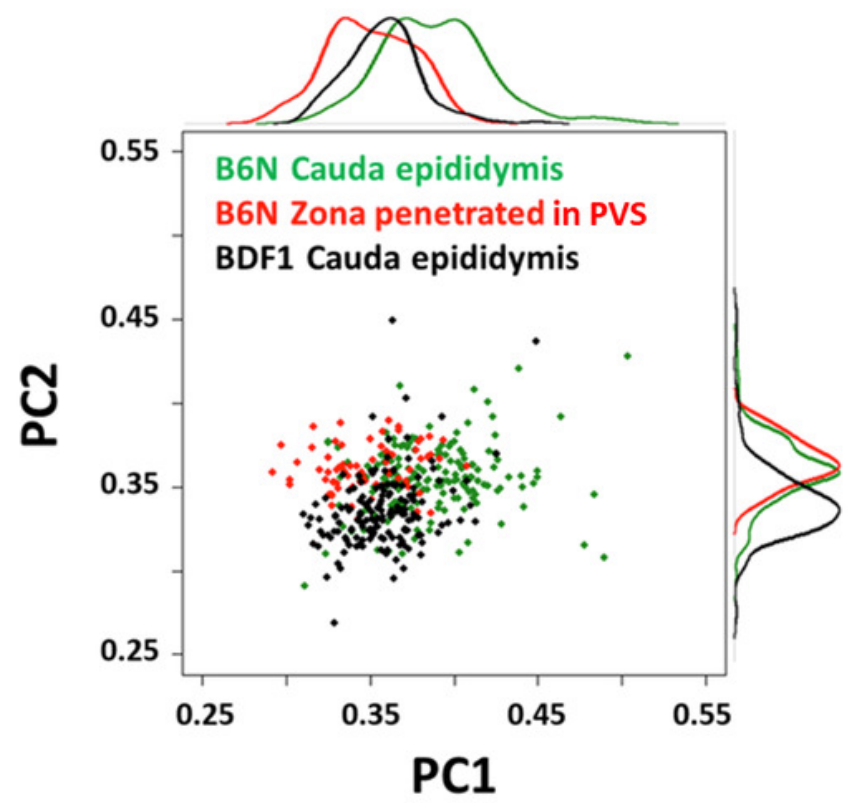




\section{Figure 3}

The sperm head aspect ratio was the largest contributor to the first principal component.

(A) Representative sperm head shapes of BDF1 (gray) and B6N (green) epididymis-isolated spermatozoa are shown with the overlapping ellipse (blue) used to calculate population mean of the aspect ratio ( $d_{1}$ in Eq. (1)). (B) A dot plot shows the aspect ratio of the heads from BDF1 and B6N spermatozoa. The error bars denote the standard deviation (SD). (C) The arrows show the eigenvectors of the PC projected into PC1-PC2 space, whose length (the square-root of the sum of the square of the factor loadings of $P C 1$ and $P C 2$ ) is greater than 0.1 , and where the dashed circle denotes the norm of the eigenvector $=1$. 
A

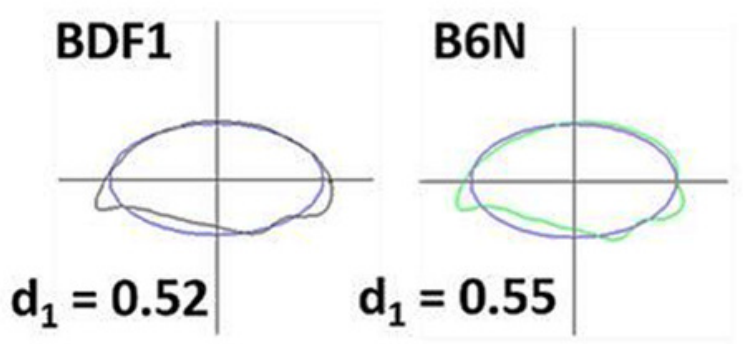

B

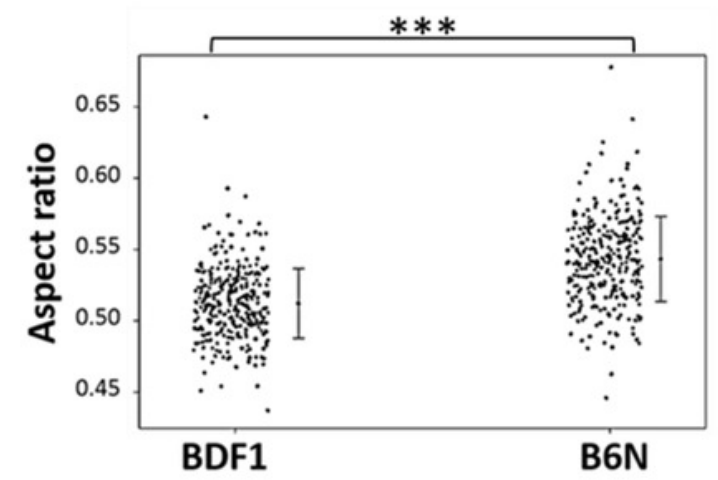

C

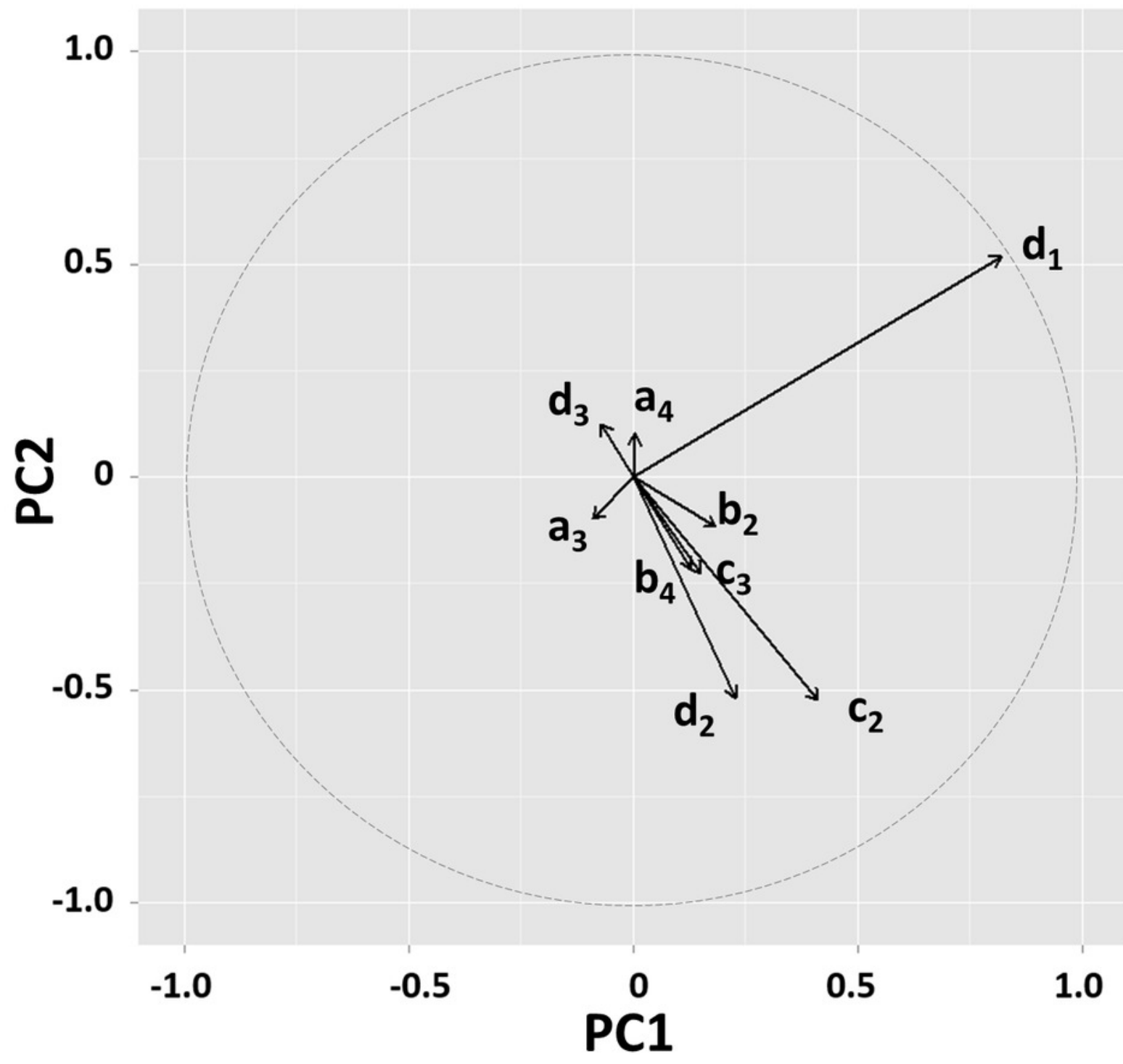




\section{Figure 4}

\section{The sperm head aspect ratio decreased during fertilization.}

(A) A box plot, with a corresponding density plot above the graph, depicts the sperm head aspect ratios, where the left/right hinge and the thick middle line represent the $25^{\text {th }} / 75^{\text {th }}$ and $50^{\text {th }}$ percentile, respectively. Spermatozoa were collected from the caput (purple), corpus (sky blue), and cauda (green) regions of the epididymis from B6N male mice $(N=5)$, or from the cauda epididymis from BDF1 male mice $(b r o w n ; N=5)$. B6N spermatozoa were also recovered from the uterus (pink) or oviducts (orange) of female mice $(N=16)$, and zona penetrated spermatozoa were recovered from the perivitelline space (PVS) of oocytes (red). The variance of the aspect ratio did not change during zona penetration (green and red; F-test, $\mathrm{P}=0.12$ ).

(B) The male and female reproductive tracts. 


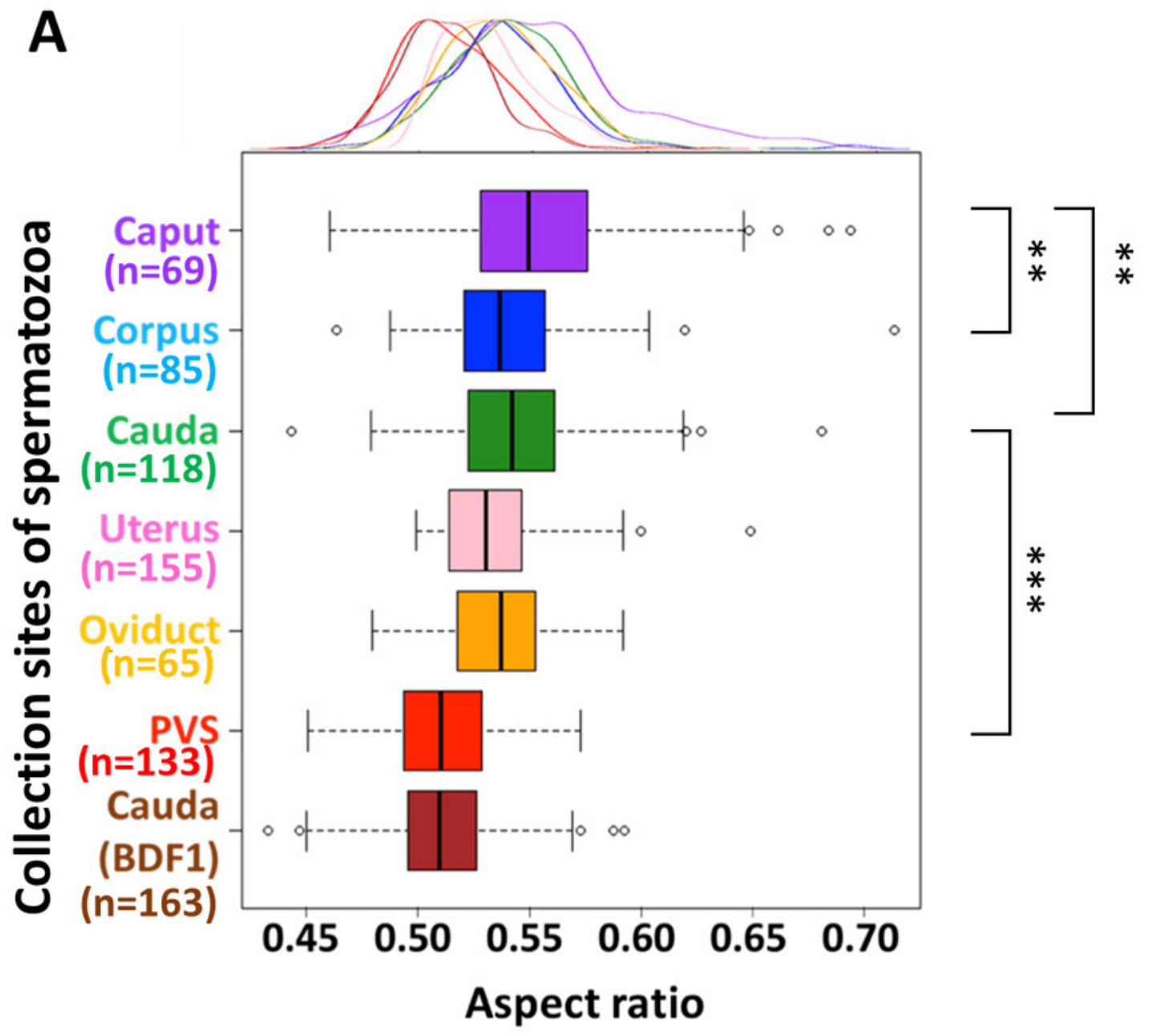

B

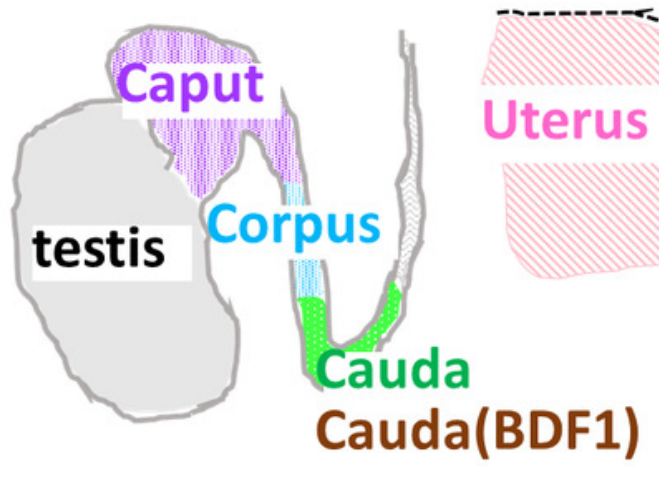

